\title{
A SEARCH FOR RAPIDLY OSCILLATING Ap STARS IN THE NORTHERN HEMISPHERE
}

\author{
Tobias J. Kreidl \\ Lowell Observatory \\ Mars Hill Rd., 1400 West \\ Flagstaff, AZ 86001 \\ U.S.A.
}

\begin{abstract}
Since 1984, efforts have been underway at Lowell Observatory to monitor Ap stars for rapid oscillations utilizing a photoelectric photometer on 0.6- to 1.8-meter telescopes. These variables, first discovered and designated as a subgroup of Ap stars by D.W. Kurtz, pulsate with periods between 4 and 15 minutes and light variations less than about $0 . \mathrm{m}_{016}$ in $\mathrm{B}$. This strongly suggests high overtone $\mathrm{p}$-mode oscillations as the cause of rapid variability. To attempt to increase the known number, Ap stars of spectral type B9 and later are being monitored. As members currently span the range A5 to FO, it is important to establish within what limits rapid oscillations are present. So far, only one new member has been found at Lowell (HD 134214), bringing the known total to eleven. The coordination of observations for known members has proved to be useful; no fewer than five rapidly oscillating Ap stars have been observed via multi-site, coordinated photometry. In this context, the status of this observing program and its future goals are presented.
\end{abstract}

\section{INTRODUCTION}

Rapidly oscillating Ap stars were first discovered and characterized as a group by Kurtz (1982). These stars are variable with periods in the range of 4 to 15 minutes and photometric amplitudes <0. $\mathrm{m}_{016}$ in $\mathrm{B}$. Their short periods are most likely due to high overtone p-modes of low degree $(l)$ and high $n(n \approx 10-40)$. These oscillations are analogous to the 5-minute variations present in our Sun.

Extensive photometric observations have been obtained for some of the presently known eleven members, ten of which have been discovered by Kurtz and one by Kreidl (1985). Several excellent reviews of the characteristics and theoretical interpretations of rapidly oscillating Ap stars have been presented recently, notably those of Kurtz (1985) and Weiss (1985).

Clearly, with so few known objects, it is highly desirable to discover new members and hence provide a larger data base. 


\section{OBSERVATIONAL EFFORTS}

Since 1984, a program has been underway at Lowell Observatory to monitor Ap stars for rapid oscillations. The primary instrumentation has been a two-channel photometer attached to Lowell's 1.1-m telescope, permitting simultaneous B,V photometry. An of fset guider is used to keep objects precisely centered in the diaphragm over long periods of time. The data acquisition system is based on a DEC LSI-11/02 computer (White and Wasserman 1984). Data are written to floppy disks, which are read in later on a VAX-11/750 for reduction and analysis. About 4 to 5 nights per month near new moon are allocated for this project. At times, Lowell's 0.6-, 0.9-, and 1.8-m telesopes have been used.

Searches have been undertaken in the northern sky, an area only somewhat covered so far since Kurtz has done most of his survey work from the Southern Hemisphere. Some work in the north has been undertaken by W. Weiss, J.A. Matthews (cf. Matthews and Wehlau 1985), and I. Elliott. The observations at Lowell have covered the spectral types B9 through early F, including some fainter Ap stars. Primary sources for candidates have been the catalogs of Bertaud and Floquet (1974) and Weiss (1984). About 20 stars to date have been monitored by the author under excellent conditions. Upon request, a list of these objects is available. It is strongly emphasized that the negative results for all but one of the objects monitored do not indicate that these objects are not pulsating; because of phase effects, the light variations due to pulsation could be invisible at times or for those stars with long rotation periods, the orientation of the magnetic pole may preclude the detection of pulsation for long periods of time ( $c f$. Kurtz 1983 for the case of $\gamma$ Equ). It is evident that the observing process is a tedious one and regularly scheduled time at an easily accessible observing site is of great benefit.

The importance of coordinating observations at multiple sites cannot be overemphasized. Interesting results have been obtained for HD 6532 (Kurtz and Kreidl 1985), HD 24712 (HR 1217) (Kurtz and Seeman 1983; Kurtz, Schneider and Weiss 1985), HD 60435 (Matthews, Kurtz and Wehlau 1986), HD 134214 (Kreidl and Kurtz 1986), and HD 128898 (Kurtz and Balona 1984).

An interesting development has been the detection of probable transient frequencies in HD 6532 and HD 60435. This raises questions regarding the stability of some of the oscillations. Multiple-site observations showing clearly the presence of such sporadic oscillations would help to establish over what time spans they tend to exist. New observations of HD 6532 (Kurtz and Cropper 1986) show how large amounts of data can have a substantial effect on the frequency analysis. An immediate benefit from multiple-site observations separated in longitude is the suppression of the $1 \mathrm{day}^{-1}$ aliasing. Even so, there are cases where observations covering an entire 24-hour cycle would be even more beneficial, such as for HD 60435.

A major effort, coordinated by D.W. Kurtz, will be undertaken in late 1986 to monitor HD 24712 (HR 1217) in order to improve the frequency analysis of this important star. 


\section{DISCUSSION}

There are clearly many puzzles in the study of rapidly oscillating Ap stars. These include the finding of uneven splitting of frequencies, the unknown lifetimes of the oscillatior modes, the question of why only few modes are excited, and the effects of the magnetic field on the pulsation (discussed to some degree by Dziembowski and Goode 1985). The phase shift, used as a good mode identifier for many types of pulsating stars (Balona and Stobie 1979), gives inconsistent results for these stars.

The temperature range in which oscillations are present is also an open question. The present upper limit is for HD 6532, which has a spectral type of $\mathrm{A} 5$ and $\mathrm{T}_{\mathrm{e}} \approx 8800 \mathrm{~K}$. It would be very revealing if all rapidly oscillating Ap stars lie within the $\delta$ Sct instability strip, making a very stong case for the $\kappa$ mechanism being responsible for pulsation.

The discovery of HD 134214 as a rapidly oscillating Ap star (Kreidl 1985; Kreidl and Kurtz 1986), is the second case (in addition to HD 60435) showing a pulsation frequency above the theoretical limit calculated by Shibahashi and Saio (1985); see also the paper by Dolez, Gough and Vauclair in this volume. Partial trapping of higher frequencies with "leakage" has been hypothesized. It is indeed questionable how well the critical frequency can be determined given all the peculiarities of Ap stars. One way to resolve the critical frequency issue would be to raise the ratio of $\mathrm{T}_{\mathrm{e}}$ to the surface temperature to 1.5 , a higher value than assumed for normal A stars. Could rapidly oscillating Ap stars have a much hotter $\mathrm{T}_{\mathrm{e}}$ than their spectral types imply? Strömgren photometry plus the $\mathrm{H} \beta$ index give no such indications. UV measurements indicate that Ap stars emit less ultraviolet flux than normal stars of the same spectral type, and furthermore, that they have cooler effective temperatures (Adelman 1985). However, the cooler Ap stars were found to be less flux deficient than the hotter Ap stars (van Dijk et al. 1976). In the IR, there is no indication of higher effective temperatures either. Shallis (1978) used the infrared flux method of Blackwell and Shallis (1977) to determine the effective temperatures and radii of a number of brighter stars, including some Ap stars. He found that for the rapidly oscillating Ap star, $\gamma$ Equ, that $\mathrm{T}_{e} \approx 7335 \mathrm{~K}, \mathrm{~d}=0.000581$ arcsec $(3.0$ $\left.+/-0.9 \mathrm{R}_{\odot}\right)$. This is indicative of a $\mathrm{T}_{\mathrm{e}}$ lower than that expected for a main sequence star of the same spectral type $F O\left(\mathrm{~T}_{e} \approx 7500 \mathrm{~K}\right)$. Due to the overabundances of rare earths, it seems more credible to assume lower surface temperatures for Ap stars compared with normal stars of the same spectral type (Shibahashi and Saio 1985) than to assume much higher effective temperatures.

\section{FUTURE CONSIDERATIONS}

There are two main observational needs that would help further our knowledge about rapidly oscillating Ap stars. The first is to search for new members so that the range of characteristics of these objects 
can be better determined. This involves large amounts of regularly scheduled telescope time on readily available instruments located on good observing sites. These factors constrain the number of astronomical establishments that can pursue such a project on a long-term basis. Lowell Observatory has proved to be able to meet all these requirements; we plan to continue the photometric monitoring of candidates at Lowell.

The second objective is to attempt to coordinate more contemporaneous observations at multiple sites. As mentioned earlier, several such undertakings have proved to aid the analysis greatly and are crucial to complete frequency analyses. We plan to continue being involved in such endeavors and encourage others to participate. Lowell's location at $+35^{\circ}$ north latitude allows access to the entire northern sky and down to about $-25^{\circ}$ declination.

With more concentrated efforts, more can be iearned about this enigmatic group of objects.

\section{REFERENCES}

Adelman, S. (1985) P.A.S.P., 97, 970.

Balona, L.A. \& Stobie, R.S. (1979) M.N.R.A.S., 189, 649.

Bertaud, Ch. \& Floquet, M. (1984) Astron. Astrophys. Suppl., 16, 71. Blackwell, D.E. \& Shallis, M.J. (1977) M.N.R.A.S., 180, 177. Dziembowski, W. \& Goode, P.R. (1985) Ap. J. Lett., 296, L27. Kreidl, T.J. (1985) I.B.V.S., No. 2739 .

Kreidl, T.J. \& Kurtz, D.W. (1986) M.N.R.A.S., 220, 313.

Kurtz, D.W. (1982) M.N.R.A.S., 200, 807.

Kurtz, D.W. (1983) M.N.R.A.S., 202, 1.

Kurtz, D.W. (1985) in Proceedings, NATO Workshop Seismology of the Sun and Distant Stars, D. Gough ed., Cambridge.

Kurtz, D.W. \& Balona, L.A. (1984) M.N.R.A.S., 210, 779.

Kurtz, D.W. \& Cropper, M.S. (1986) preprint.

Kurtz, D.W. \& Kreidl, T.J. (1985) M.N.R.A.S., 216, 987.

Kurtz, D.W., Schneider, H. \& Weiss, W.W. (1985) M.N.R.A.S., 215, 77.

Kurtz, D.W. \& Seeman, J. (1983) M.N.R.A.S., 205, 11.

Matthews, J.A. \& Wehlau, W. (1985) P.A.S.P., 97, 841.

Matthews, J.A., Kurtz, D.W. \& Wehlau,W. (1986) Ap. J., 300, 348.

Shallis, M. (1978) in Ap-Stars in the Infrared, p. 47, W.W. Weiss,

T.J. Kreidl eds., Vienna.

Shibahashi, H. \& Saio, H. (1985) Pub. astr. Soc. Japan, 37, 245.

van Dijk, W., Kerssies, A., Hammerschlag-Hensberge, G. \& Wesselius, P.R.

(1976) Astron. Astrophys. Suppl., 66, 187.

Weiss, W.W. (1984) Vienna Internal Report, 1984/1.

Weiss, W.W. (1985) in Proceedings, I.A.U. Coll. No. 90, in press.

White, N.M. \& Wasserman, L.H. (1984) P.A.S.P., 96, 667. 